\title{
INTEGRATED NAVIGATION - FLIGHT CONTROL SYSTEM OF GUIDED PROJECTILES AND BOMBS
}

\author{
Ryszard Vogt, MirosŁaW Adamski \\ Polish Air Force Academy, Faculty of Aviation and Aeronautics, Dęblin, Poland \\ e-mail: sekretariatdziekana@wsosp.deblin.pl

\section{ROBERT GŁĘBOCKI} \\ Warsaw University of Technology, Faculty of Power and Aeronautical Engineering, Warsaw, Poland
}

Integrated navigation and flight control systems have found a widespread use in projectiles. Currently, due to their advantages, they begin to be used more frequently in airplanes and helicopters. The paper presents one of the most advanced solutions of the integrated navigation - the flight control system designed for guidance of projectiles and aerial bombs.

Keywords: navigation, flight control system, bomb

\section{The development of the system}

The division of the control system adopted in the block diagram (Fig. 1) into the navigation and flight control subsystems is contractual. Technical implementation of signal processing has been integrated (Koruba and Ładyżyńska-Kozdraś, 2010).

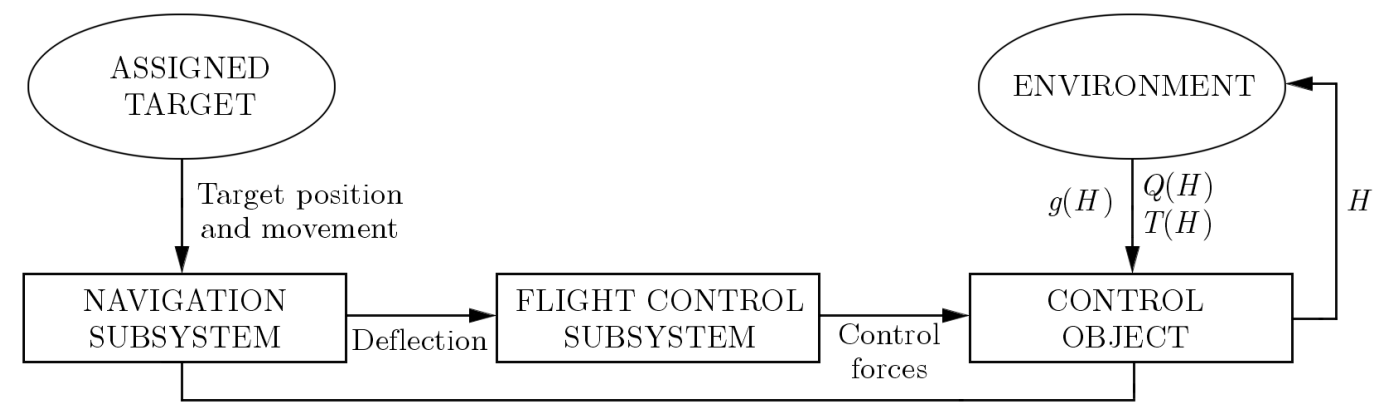

Fig. 1. Block diagram of the guidance system of the object to the target (source: own data)

The most important factor for the fulfillment of such requirements is to adopt the following system solutions:

- Integration of navigation and flight control functions, which, among others, eliminates the necessity of performing independent measurements as well as reduces control procedures and increases performance of the system;

- The target tracking process and control signals production take place in the coordinate system pertaining to the object;

- Limitation of the amount of flight control channels in three-dimensional space to a single-dimensional one;

- Change of the flight direction with the use of properly arranged and coordinated rocket engines, acting upon centre of gravity of the object (Grzesik, 2011). 


\section{Description of the system performance}

The guidance system is implemented in a system of coordinates pertaining to the rotary projectile, and is single channel. The characteristic feature of the system is the lack of moving parts, which, among others, ensures high operational reliability.

The range of navigation features includes:

- Programmed initiation of target tracking process;

- Optimization of the flight control initiation time;

- Measurement by a tracking head of the target observation deflection line from the projectile axis - it is so called angular deflection (Fig. 2);

- Determination of the trajectory aimed at reaching the target and processing of the deflection signal to the control signal $K$, which ensures the projectile flight to the target along the predetermined trajectory (Głębocki and Vogt, 2007).

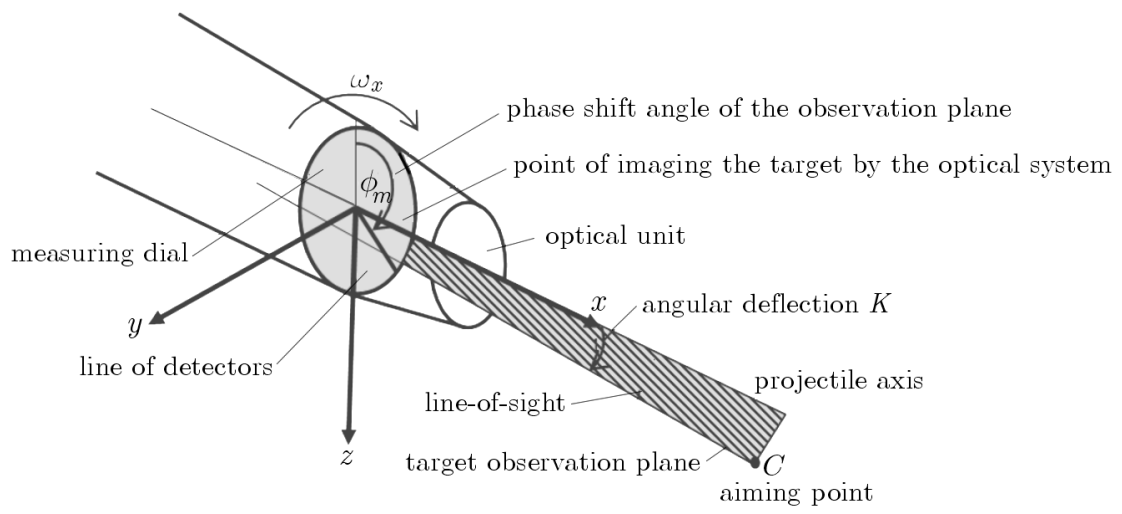

Fig. 2. Principle of operation of the head measuring system ( $K$ - angular deflection, $E-$ pulse deflection signal, $\phi_{m}$ - angle of inclination of the target measurement plane, the so-called angle of target location (source: own data)

In the elaborated guidance method above, the issue of navigation, i.e. determination of the trajectory aimed at reaching the target as well as formation of the control signal have been solved together. Due to the fact that the tracking head detector is rigidly mounted in the spinning projectile and due to resignation from the gyroscopic reference system, the deflection is measured with regard to the projectile axis (Fig. 3). The importance of the method is striving to ensure that the longitudinal axis of projectile $X_{1}$ throughout the controlled flight is directed to the target. Therefore, this method is simply trying to minimize the angular deflection $K$ (Fig. 3).

\subsection{Tracking head}

To measure the target position, a one-dimensional linear detector is used, which is rigidly attached to the spinning projectile (Fig. 2). Thus, the target measurement with regard to the projectile takes place in polar coordinates. Because the detector is positioned radially on the measuring dial spinning with the projectile, signal $E$ with angular deflection with regard to the projectile axis is received at the moment in which the detector is located in the target measurement plane, inclined at the angle $\phi_{m}$. Therefore, the deflection signal $E$ is a series of pulses (Fig. 4) occurring at a frequency $\omega$ equal to the frequency of the projectile rotation around the longitudinal axis $X$. Because of the segmented structure of the detector, the value of the pulse signal of the deflection $E$ abruptly changes with the deflection angle $K$ (Fig. 3). Due to the lack of a gyroscopic reference system, subsequent moments $t_{n}$ (where $n=0,1,2,3, \ldots$ ), in which 


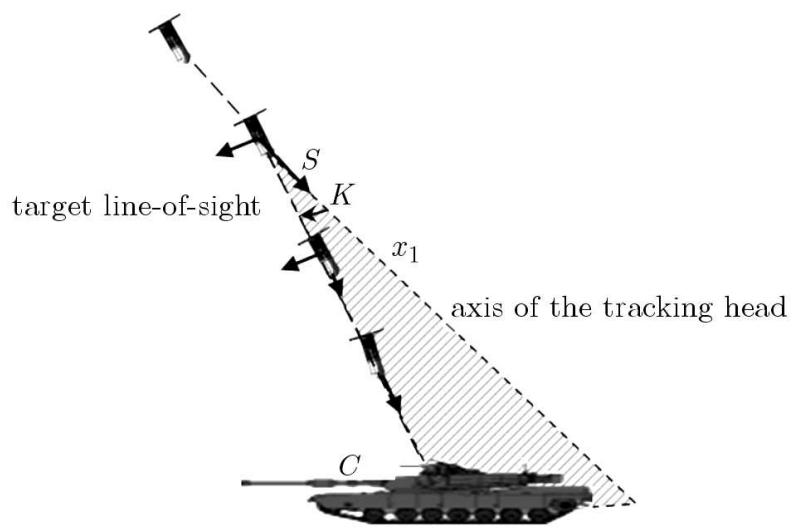

Fig. 3. A simple scheme of the guidance (source: own data)

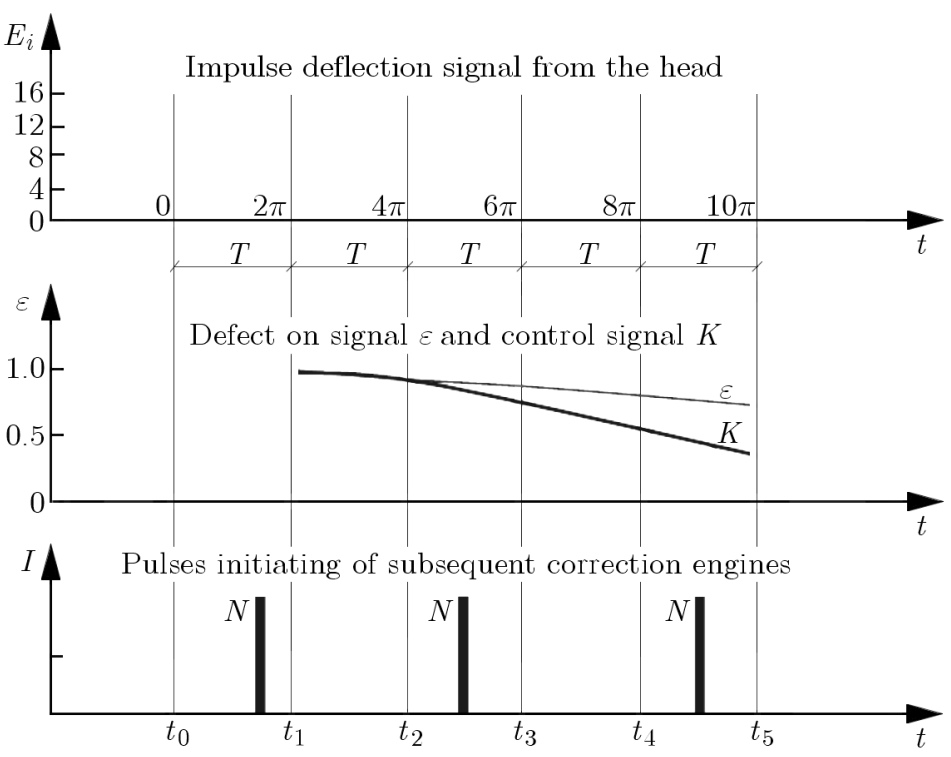

Fig. 4. Course of the angular deflection and pulse deflection signal registered by the head (source: own data)

the signal occurs, are subsequent time reference points of the whole control process reference (Stefański and Koruba, 2012).

\subsection{Formation of the deflection signal}

To permit the projectile guidance on the pulse target, the deflection signal $E$ is appropriately processed for the continuous signal $\varepsilon$ (Fig. 4) and is subject to:

- Linearization, i.e. processing for continuous signal;

- Forecasting - determination of the course with corresponding anticipation of the course of time;

- Noise filtering caused by time discontinuities of signal $E$ (Fig. 4) and by oscillation of the projectile in the range of the angle of attack and sideslip (Głębocki and Vogt, 2000).

A method of polynomial approximation has been used to linearization and forecasting.

The main drawback of the simple guidance method is that the implemented flight trajectory requires high gravity loads to guide onto targets that move at a greater speed. In order to reduce these gravity loads, the method has undergone an appropriate modification. The essence of this change is to minimize, while guiding, not only the angle but also the angular velocity 
of the deflection angle. In addition, to ensure greater stability of the flight control process, an additional noise filtering is introduced by the introduction of a first order inertia element. Hence, the control law takes form typical for the PD-type control

$$
T \frac{d K}{d t}+K=k_{a}\left(\varepsilon+T_{f} \frac{d \varepsilon}{d t}\right)
$$

where $K$ is the control signal at the output of navigation and control units; $T$ - time constant of the unit; $k_{a}$ - gain; $T_{f}$ - differentiating constant.

The parameters $T, T_{f}, k_{a}$ depend on dynamic properties of the projectile as well as on target motion and position with regard to the launcher.

In the adopted solution, the flight control is performed by means of rocket correction engines arranged radially around the target centre of gravity (of projectile, bomb). Starting a single engine generates the force pulse headed in a perpendicular manner to the axis of symmetry and along a straight line passing through its centre of gravity (Figs. 3 and 6).

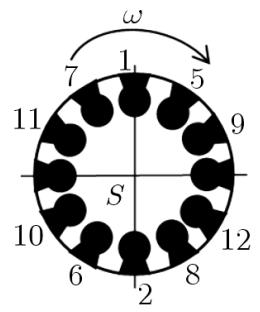

Fig. 5. Location of correction engines around the bomb axis (source: own data)

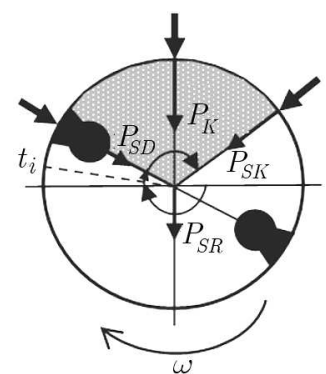

Fig. 6. Principle of the projectile guidance using rocket correction engines. The grey area represents the operation time of the correction engine (source: own data)

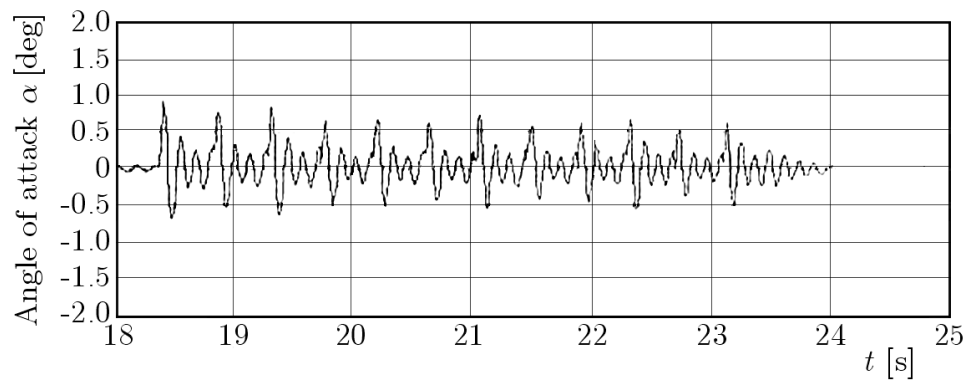

Fig. 7. Changes in the angle of attack at a multiplicity of $N=3$ (source: own data)

The engine activation directly affects the velocity vector change of the bomb flight with respect to both the direction as well as the magnitude. On the basis of the measurement of the bomb position with regard to the designated target point and to the predetermined flight trajectory, the time and direction of pulses correcting the flight path are worked out, and then the signals for pulse rocket thrusters are initiated (Głębocki and Vogt, 2000). 
The engines are arranged radially around the centre of gravity. They give off single control pulses headed in a perpendicular manner to the main projectile axis. The function which initiates the engine start-off depends on the value of the control signal, on the phase position of the target as well as on the angular position of the projectile spinning motion (Figs. 2 and 6). The spatial flight of the target, with a single-channel control, is possible thanks to swirling motion of the target and to starting at the moment corresponding to the angle $\phi_{m}$ of the phase target position (Fig. 2).

\section{Conclusions}

Single-channel pulse direct flight control allows achieving the required control quality with appropriate selection of engine start-off algorithms as well as with dynamic stability of the projectile. Hence, the stability of the directly controlled projectiles can be arbitrarily large. The engine start-off algorithms are highly complex. In their designation, one should take into account such an order of projectile launching at which the dynamic imbalance of the projectile will be minimal. These algorithms must ensure the required flight control quality, among others, thanks to proportionality of the average control effect to the value of the control signal (Głębocki and Vogt, 2007).

Properties of the projectile controlled by a set of impulse engines:

a) Pulse control is limited in the number of correction pulses.

b) The reaction is much faster, a change in the direction does not require inclination of the projectile.

c) During the direct control, there appear much smaller angles of attack and sideslip.

d) Pulse implementing systems do not have moving mechanisms complicating the structure and increasing demand for energy.

e) Requirements for the projectile aerodynamics are reduced.

f) The projectile control does not depend on flight velocity, which constitutes the greatest advantage of targets of this class.

\section{References}

1. GŁębocki R., Vogt R., 2000, Impulse control of anti-tank missile, NATO-RTO Meeting Proceeding, Brunschwig

2. GŁęBocki R., Vogt R., 2007, Guidance systems of smart mortar missile, The Archive of Mechanical Engineering, LIV, 1

3. GRZESIK N., 2011, Zaawansowane systemy uzbrojenia lotniczego. Budowa i zastosowanie, WSOSP, Dęblin

4. Koruba Z., Ładyżý́sKa-Kozdraś E., 2010, The dynamic model of combat target homing system of the unmanned aerial vehicle, Journal of Theoretical and Applied Mechanics, 48, 3, $551-566$

5. Stefański K., Koruba Z., 2012, Analysis of the guiding of bombs an ground targets using a gyroscope system, Journal of Theoretical and Applied Mechanics, 50, 4, 473-485 